\title{
Mutation screening using formalin-fixed paraffin-embedded tissues: a stratified approach according to DNA quality
}

\author{
Francesco Cucco ${ }^{1} \cdot$ Alexandra Clipson ${ }^{1} \cdot$ Hannah Kennedy ${ }^{1} \cdot$ Joe Sneath Thompson $^{1} \cdot$ Ming Wang $^{1}$ • \\ Sharon Barrans ${ }^{2} \cdot$ Moniek van Hoppe $^{2} \cdot$ Eguzkine Ochoa Ruiz $^{1} \cdot$ Josh Caddy ${ }^{3} \cdot$ Debbie Hamid $^{3} \cdot$ Thomas Cummin $^{4}$. \\ Cathy Burton ${ }^{2} \cdot$ Andrew J Davies ${ }^{4} \cdot$ Peter Johnson ${ }^{4} \cdot$ Ming-Qing Du (i) ${ }^{1,5}$
}

Received: 7 February 2018 / Revised: 28 March 2018 / Accepted: 29 March 2018 / Published online: 16 May 2018

(c) United States \& Canadian Academy of Pathology 2018

\begin{abstract}
DNA samples from formalin-fixed paraffin-embedded tissues are highly degraded with variable quality, and this imposes a big challenge for targeted sequencing due to false positives, largely caused by PCR errors and cytosine deamination. To eliminate false positives, a common practice is to validate the detected variants by Sanger sequencing or perform targeted sequencing in duplicate. Technically, PCR errors could be removed by molecular barcoding of template DNA prior to amplification as in the HaloPlexHS design. Nonetheless, it is uncertain to what extent variants detected using this approach should be further validated. Here, we addressed this question by correlating variant reproducibility with DNA quality using HaloPlexHS target enrichment and Illumina HiSeq4000, together with an in-house validated variant calling algorithm. The overall sequencing coverage, as shown by analyses of 70 genes in 266 cases of large B-cell lymphoma, was excellent (98\%) in DNA samples amenable for PCR of $\geq 400 \mathrm{bp}$, but suboptimal (92\%) and poor (80\%) in those amenable for PCR of $300 \mathrm{bp}$ and $200 \mathrm{bp}$ respectively. By mutation analysis in duplicate in 93 cases, we demonstrated that 20 alternative allele depth (AAD) was an optimal cut-off value for separating reproducible from non-reproducible variants in DNA samples amenable for PCR of $\geq 300 \mathrm{bp}$, with $97 \%$ sensitivity and $100 \%$ specificity. By cross validation with a previously established targeted sequencing protocol by Fluidigm-PCR and Illumina MiSeq, the HaloPlexHS protocol was shown to be highly sensitive and specific in mutation screening. To conclude, we proposed a stratified approach for mutation screening by HaloplexHS and Illumina HiSeq4000 according to DNA quality. DNA samples with good quality ( $\geq 400 \mathrm{bp}$ ) are amenable for mutation analysis with a single replicate, with only variants at 15-20 AAD requiring for further validation, while those with suboptimal quality $(300 \mathrm{bp}$ ) are better analysed in duplicate with reproducible variants at $>15$ AAD regarded as true genetic changes.
\end{abstract}

These authors contributed equally: Francesco Cucco, Alexandra Clipson.

Electronic supplementary material The online version of this article (https://doi.org/10.1038/s41374-018-0066-z) contains supplementary material, which is available to authorized users.

Ming-Qing Du

mqd20@cam.ac.uk

1 Department of Pathology, University of Cambridge, Cambridge, UK

2 HMDS, Leeds Cancer Centre, Leeds Teaching Hospitals NHS Trust, Leeds, UK

\section{Introduction}

Next generation sequencing (NGS) has made an unprecedented contribution to discoveries in the biomedical research field. One of its many applications is targeted sequencing to screen mutations in a panel of interesting genes. In view of the rapid discovery in cancer research and huge impact from mega genome sequencing initiatives such

3 Southampton Clinical Trials Unit, University of Southampton, Southampton, UK

4 Cancer Research UK Clinical Centre, University of Southampton, Southampton, UK

5 Department of Histopathology, Addenbrooke's Hospital, Cambridge University Hospitals NHS Foundation Trust, Cambridge, UK 
as the $100 \mathrm{k}$ genome project, it is imperative to establish highly sensitive, specific and also robust targeted sequencing protocol that is amenable to degraded DNA from formalin fixed paraffin embedded (FFPE) tissues/cells, allowing translational research as well as routine clinical application. There are several target enrichment methods available, based on hybridisation capture (Agilent SureSelect or NimbleGen SeqCap products), PCR (Fluidigm Access Array PCR, RainDance technology) or a combination of both hybridisation capture and PCR (HaloPlexHS). The enriched target sequence library can then be sequenced by an Illumina or Ion Torrent platform. Among these different approaches, PCR-based targeted enrichment is commonly used due to its easy application to minute amounts of template DNA.

There are many issues associated with targeted sequencing, particularly when using DNA samples from FFPE tissues. One major issue is the uncertainty whether the variants detected are true genetic changes or false positives due to PCR/sequence errors, poor DNA quality and deamination of cytosine. Treatment of FFPE tissue DNA with uracil glycosylase can significantly reduce false positives resulting from deamination of cytosine, but this has little or no effect for deamination-induced change at 5-methyl cytosine and $\mathrm{CpG}$ dinucleotides [1-3]. A more common practice is to further verify the variants detected by an independent method such as conventional Sanger sequencing or an alternative targeted sequencing protocol [4]. Other major issues for targeted sequencing are suboptimal performance of variant calling algorithms particularly for indels, and a lack of an experimentally established cut-off value, for example variant allele depth and frequency, for highly sensitive and specific detection of somatic mutations [5].

In a recent study, we have established a targeted sequencing protocol by Fluidigm Access Array PCR and Illumina MiSeq sequencing together with an in house validated variant calling pipeline that was optimised against a large number of various known mutations [6]. False positives were eliminated by performing the targeted sequencing and data analyses in duplicate with only the variants, which appeared in both replicates and above the experimentally defined cut-off value of alternative allele frequency (AAF), being regarded as true genetic changes [6]. Detailed analyses of false positives revealed their distinct nature and origins between high molecular weight DNA from fresh frozen tissues and degraded DNA from FFPE tissues. For high molecular weight DNA, the majority of false positives are derived from PCR/sequencing errors, while for FFPE tissue DNA, the false positives are from both $\mathrm{PCR} /$ sequencing errors and cytosine deamination, which is caused by tissue formalin fixation and storage [2, 6-9].
The more recent HaloPlexHS target enrichment design incorporates a molecular barcode in hybridisation probes, and this allows removal of PCR errors during sequence data analysis. Apart from this, the HaloPlexHS target enrichment also offers several other advantages including easy probe design, high flexibility in the number of genes to be investigated, and a streamlined experimental protocol. Nonetheless, it remains to be investigated whether mutation analysis can be reliably performed in a single replicate with DNA samples from FFPE tissues by the HaloPlexHS target enrichment protocol. In the present study, we have optimised a protocol for high throughput mutation screening by HaloPlexHS target enrichment and Illumina sequencing, and established a practical strategy for reliable mutation detection using DNA samples from FFPE tissues. The strategy allowed stratification of DNA samples into different protocols of mutation analyses according to their quality, with good quality DNA investigated in a single replicate, while sub-optimal quality DNA was analysed in duplicate.

\section{Materials and methods}

\section{Tumour materials and DNA extraction}

FFPE lymphoma specimens were retrieved from 266 cases of diffuse large B-cell lymphoma (DLBCL) enrolled to the REMoDL-B and MaPLe trials. Local ethical guidelines were followed for the use of these tissue materials for research with the approval of the ethics committees of the involved institutions.

Haematoxylin and eosin slides were reviewed and tumour rich areas $(>40 \%)$ in each specimen were isolated by crude microdissection for DNA extraction. DNA was extracted using the QIAamp DNA Micro Kit (QIAGEN, Crawley, UK) and quantified using a Qubit $^{\circledR}$ Fluorometer (Life Technologies, UK).

\section{Assessment of DNA quality by conventional PCR}

This was performed by PCR of variably sized genomic fragments using $2 \mathrm{ng}$ template DNA in a $10 \mu \mathrm{l}$ reaction mixture for 40 cycles under a standardised protocol as described previously [6].

\section{Targeted sequencing by Fluidigm multiplex PCR and Illumina MiSeq sequencing}

This was used to investigate mutations in 22 genes in 60 cases of DLBCL as described previously [6]. Each DNA sample was simultaneously investigated in duplicate. Briefly, $50 \mathrm{ng}$ genomic DNA was used for preamplification 
and Fluidigm Access Array PCR, followed by barcoding and Illumina MiSeq sequencing. Variants were identified using an in-house developed and validated variant caller python program [6]. After filtering baseline sequence errors and germline changes through SNP database search, novel variants seen in both replicates of the same sample were recorded and those above $10 \%$ AAF were regarded as true changes as defined previously [6].

\section{Gene panel and target enrichment by HaloPlexHS}

A total of 70 genes ( $205 \mathrm{~kb}$ sequence) that are recurrently mutated in aggressive B-cell lymphomas were included in the HaloPlexHS target enrichment design, and they included the 22 genes that were investigated by Fluidigm multiplex PCR and Illumina MiSeq sequencing as outlined above [6]. The HaloPlexHS target enrichment design incorporates molecular barcodes in the hybridisation probes, thus allowing the removal of PCR errors during sequence data analysis (Agilent Technologies). HaloPlexHS target enrichment was performed essentially according to the manufacturer's instructions for FFPE tissue samples. Briefly, $100 \mathrm{ng}$ of genomic DNA was digested with restriction enzymes, and hybridized to the above customised HaloPlexHS probe library. The probe-target DNA hybrids were ligated and circularized with HS DNA ligase, then purified with streptavidin beads and finally amplified by PCR.

\section{Library purification and Illumina sequencing}

The above amplified target library was purified twice using AMPure XP beads (Beckman Coulter, Pasadena, CA) to remove fragments below the expected target size. The purified target library from each sample was then validated and quantified using the 4200 TapeStation (Agilent Technologies), and pooled with appropriate adjustment according to their concentration. The pooled libraries were then sequenced on one of the following Illumina platforms: MiSeq $(2 \times 250 \mathrm{bp}$ end sequencing protocol), HiSeq2500 (Rapid Run Mode $2 \times 150$ bp end sequencing protocol), or HiSeq4000 $(2 \times 150 \mathrm{bp}$ end sequencing protocol).

\section{Variants calling and data analysis}

Demultiplexing and conversion from bcl to fastq were performed using bcl2fastq v2.19, followed by read trimming and adaptor sequence removal with SurecallTrimmer from the Agilent Genomics NextGen Toolkit v4.0.1 (AGeNT). The reads were aligned to hg38 using bwa mem v0.7.17 and deduplication was carried out using the AGeNT LocatIt tool. The resultant sam files were converted to bam with samtools v1.3.1, which was also used for sorting and indexing of bam files [10].
For SNV detection, bam files were processed using a pipeline based on GATK v3.6 best practices including indelrealigned and recalibration steps. The calling variant was run using UnifiedGenotyper with 10,000 to prevent downsampling [11]. As GATK was unable to call SNVs at $<8 \%$ AAF reliably, MuTect2 was additionally employed for detection of hotspot mutations at low AAF values. Indel detection was separately carried out on the recalibrated bam files using Pindel v0.2.5 [12], which allowed detection of indels as low as $2 \% \mathrm{AAF}$.

Variant call files were concatenated to produce one library vcf each for the SNV and Indel pipelines. These library files were then filtered using a combination of veftools v0.1.15 and bedtools v2.25 for read depth, quality score, and known PCR/sequence artefacts [13, 14]. Further filtering was accomplished using an in-house script to remove variants in intronic regions outside essential splicing sites, SNPs with a minor allele frequency $\geq 1 \%$ and synonymous changes. The resulting novel variants were further scrutinised by reviewing the bam file to eliminate any potential $\mathrm{PCR} /$ sequence artefacts.

The above in house variant calling pipeline was optimised and validated using two virtual sequence libraries containing a large number of various known somatic mutations: one contained lymphoma associated mutations (60 SNVs, 19 indels and 6 splicing variants), while the other included brain tumour associated mutations (5 SNVs, 11 indels), largely unrelated to the current study.

\section{Results}

\section{Optimisation of experimental protocols}

In the initial study, a series of testing experiments were performed in duplicate to optimise the experimental protocols. First, various amounts of template DNA $(50,100 \mathrm{ng})$ from representative FFPE DLBCL tissue specimens (DNA samples amenable for PCR of up to $200 \mathrm{bp}(n=1), 300 \mathrm{bp}$ $(n=5)$ and $400 \mathrm{bp}(n=3)$ genomic fragment) with known mutations in 22 of the 70 genes investigated were used for HaloPlexHS target enrichment, followed by Illumina sequencing. Based on the coverage and depth of sequencing, $100 \mathrm{ng}$ DNA was considered as the minimal optimal amount of template DNA for the targeted sequencing (Fig. S1A). Second, we compared the sequence coverage among different Illumina sequencing platforms, namely MiSeq, HiSeq2500 and HiSeq4000 with compatible amounts of target enrichment library according to their sequencing capacity. The results showed that HiSeq4000 yielded the highest overall sequence coverage and depth reads, much better than HiSeq2500 based on analysis of 80 samples respectively (Fig. S1B). Hence, Illumina 
HiSeq4000 was chosen for all of the subsequent experiments, with all of the data presented below being derived from optimised HaloPlexHS target enrichment and Illumina HiSeq4000 sequence unless otherwise specified.

\section{Impact of DNA quality on sequence coverage and variant reproducibility}

A total of 266 FFPE DLBCL tissue specimens were investigated by targeted sequencing of 70 genes using HaloPlexHS target enrichment and Illumina HiSeq4000. As expected, the quality of DNA samples had a major impact on the target library quantity, overall sequence coverage as well as the read depth, with better quality of DNA samples clearly showing higher performance of these parameters (Fig. 1A-D). In addition, the DNA samples amplifiable for $\geq 400$ bp genomic fragments showed a much lesser extent of variation in their sequence coverage than those only amplifiable for up to $300 \mathrm{bp}$ (Fig. 1D). There was also a correlation between the depth reads and the amplicon sizes, with higher depth reads in smaller rather than larger sized amplicons (Fig. S2).

To examine whether DNA quality had any impact on the reproducibility of variant detection, we next focused on the 93 cases where duplicate experiments starting from input DNA were carried out. The variants identified by the in house variant calling pipeline were filtered to remove variants that were deemed unacceptable: $<50$ total read depth (TD), $<5$ alternative allele read depth (AAD), $<2 \% \mathrm{AAF}$, as well as known PCR/sequencing artefacts. The resulting novel variants were interrogated between the two replicates, and recorded as reproducible or non-reproducible changes, with the latter group being most likely false positives (Fig. S3). As shown in Fig. 1E, the proportion of non-reproducible variants clearly depended on the quality of DNA, being much higher in DNA samples amplifiable for $\leq 300 \mathrm{bp}$ than those amplifiable for $\geq 400 \mathrm{bp}$ genomic fragment. Importantly, a high proportion of these non-reproducible variants had a high AAF, limiting its value to separate reproducible from nonreproducible changes (Fig. 1F).

\section{Determining cut-off parameters for reliable variant detection}

The HaloPlexHS target enrichment design incorporates a molecular barcode, allowing removal of PCR duplicates, thus PCR errors. In view of this, the number of reads that bear novel variants, i.e., alternative allele depth (AAD), would represent the copy number of "mutant" template that are successfully captured and sequenced. Theoretically, this would be a good parameter to distinguish true genetic changes from false positives as the higher the $\mathrm{AAD}$, the higher the probability of a variant originating from template DNA rather than experimental artefact. As expected, all non-reproducible variants in DNA samples amplifiable for $\geq 400 \mathrm{bp}$ or up to 300 bp genomic fragment were at low AAD values with mean plus 2 SD being $<15$ in both groups (Fig. 2A, B). To ensure highly specific mutation detection, we used $20 \mathrm{AAD}$ as the cut-off value for dichotomy between reproducible and nonreproducible variants. This allowed detection of $97 \%$ reproducible variants with $100 \%$ specificity. As all nonreproducible variants are below this cut-off value, theoretically a single replicate could be sufficient for reliable mutation detection if this threshold proved to be highly efficient and specific. To verify this, we next examined the concordance in mutation detection between HaloPlexHS/Illumina HiSeq and Fluidigm/Illumina MiSeq approaches.

Non-reproducible variants in DNA samples amplifiable for only up to $200 \mathrm{bp}$ genomic fragment were rather dispersed, with a high proportion showing relatively high AAD values (Fig. 2C).

\section{Validation of mutation detection by HaloPlexHS/ Illumina HiSeq sequencing}

Among the cases investigated by HaloPlexHS /Illumina HiSeq4000, 60 (22 in duplicate and 38 in a single replicate) were also investigated for mutations in 22 genes by Fluidigm Multiplex PCR/Illumina MiSeq in duplicate. The novel variants that were considered as true genetic changes were identified independently by their respective protocols and thresholds [6], and then compared between the two different targeted sequencing approaches.

Within the 22 cases that were investigated in duplicate by both Fluidigm and HaloPlexHS approaches, 60 and 61 novel variants were identified in the common region of 22 genes covered by these methods respectively, with 58 variants being mutually detected by both methods (Fig. 3A). The two variants detected by Fluidigm but not HaloPlexHS approach were found by the HaloPlexHS approach but not called as both variants were at the end of their amplicon, while the three variants detected by HaloPlexHS, but not Fluidigm approach were due to low AAF value.

Of the 38 cases that were investigated in duplicate by the Fluidigm, but a single replicate by the HaloPlexHS approach, 96 and 98 novel variants were identified in the common region of 22 genes covered by these methods respectively, with 91 variants being mutually detected by both methods (Fig. 3B). The five variants detected by Fluidigm but not the HaloPlexHS approach were due to suboptimal AAD (6 and 12) in 2, low AAF in 2, and at the end of its amplicon in 1 , while the seven variants detected by HaloPlexHS, but not the Fluidigm approach were due to low AAF value.

Taken together, the above findings indicate that the target sequencing protocol by HaloPlexHS/Illumina HiSeq was 
A)

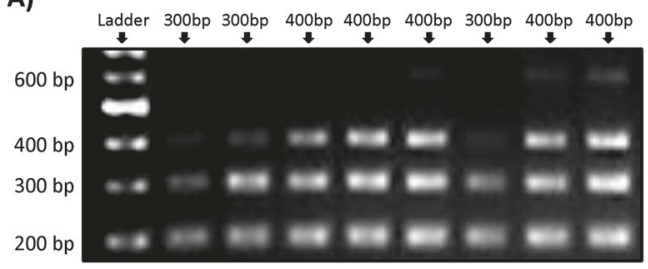

B)

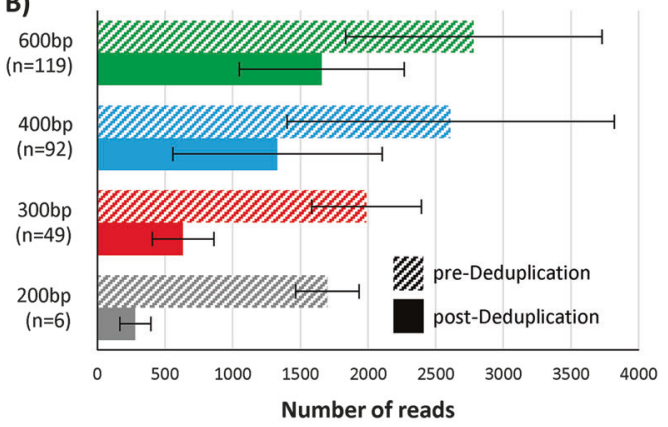

C)

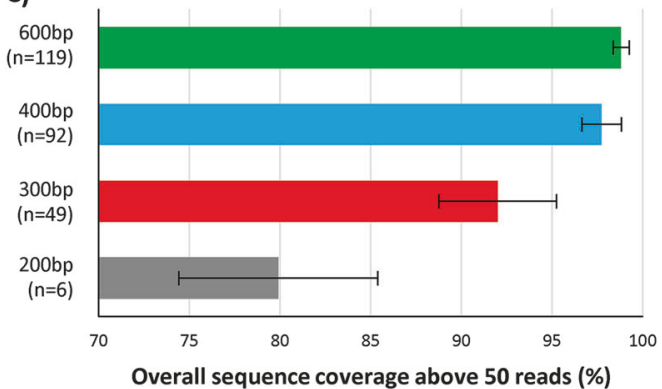

E)

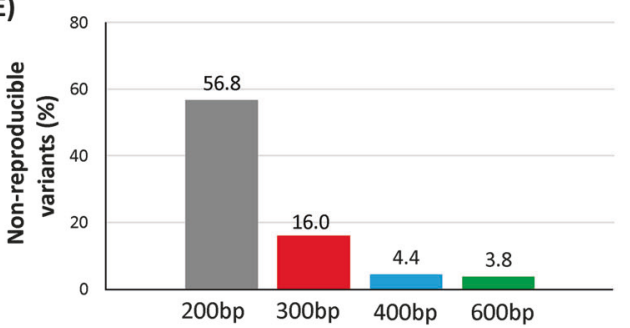

D)

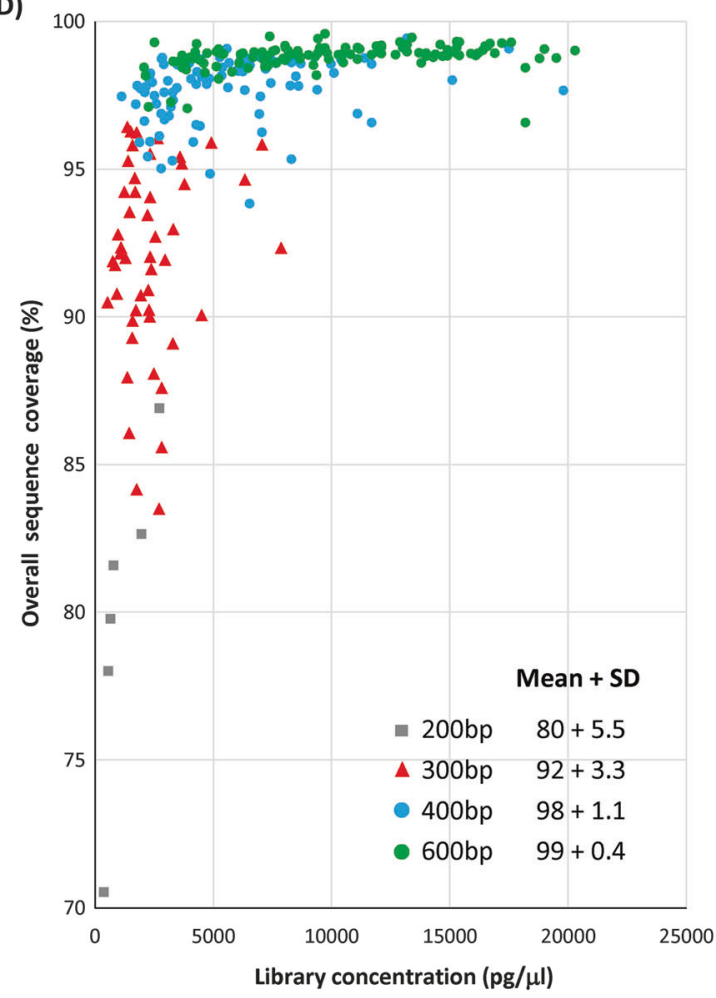

F)

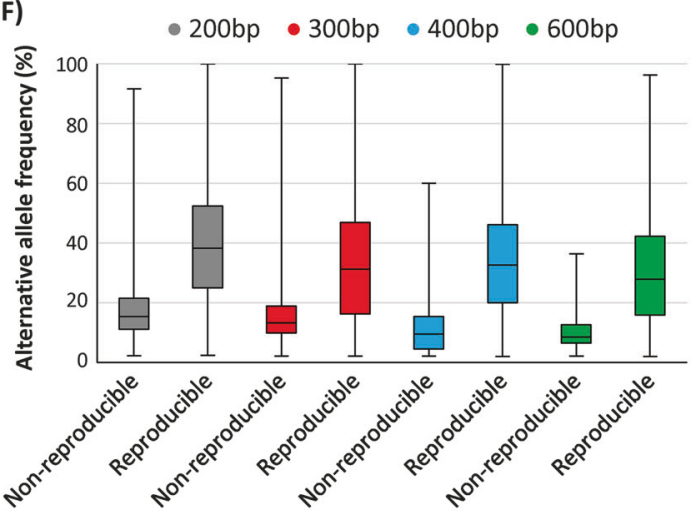

sequencing coverage, while those that support PCR of only up to 300 bp genomic fragment generate much lower quantity of target sequence libraries and suboptimal sequencing coverage. Mean and SD of sequencing coverage are provided according to DNA quality. E) Extent of non-reproducible variants according to DNA quality. The analysis is based on variants after initial filtering to remove those deemed unacceptable: $<50$ total read depth, $<5$ alternative allele read depth, $<2 \%$ alternative allele frequency, as well as known PCR/ sequencing artefacts. F) Comparison of alternative allele frequency (AAF) between reproducible and non-reproducible variants according to DNA quality. There is considerable overlap in AAF between reproducible and non-reproducible variants regardless of their DNA quality, hence limiting its value in identification of true genetic changes. 
A) DNA $(400 / 600 \mathrm{bp}, \mathrm{n}=66)$

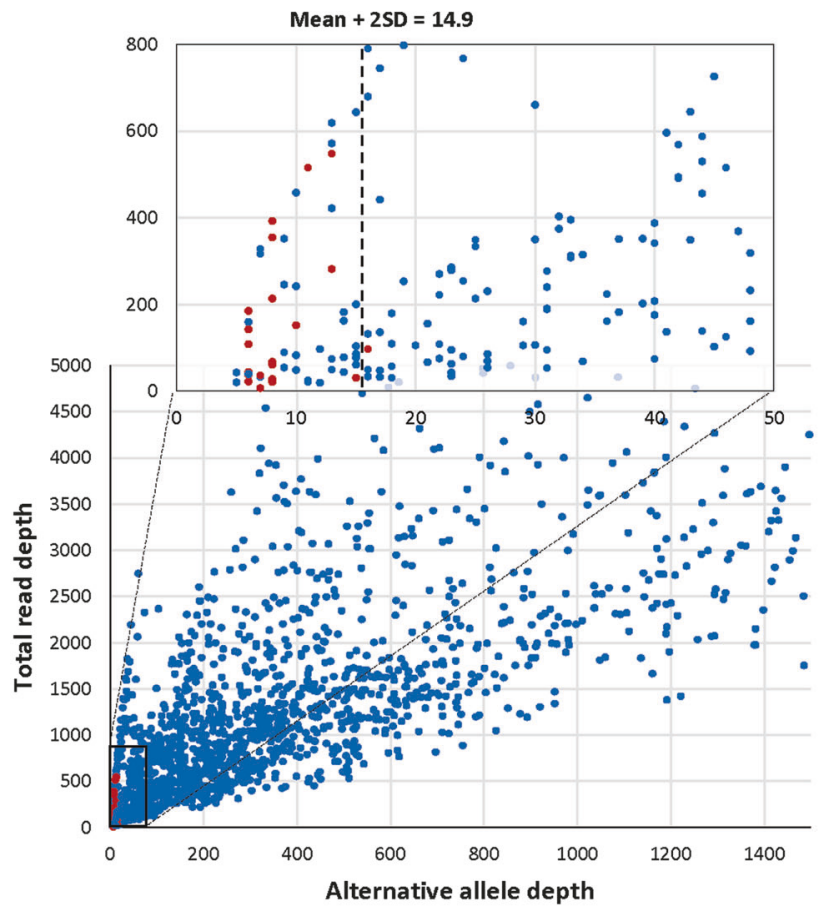

C) DNA (200bp, $\mathrm{n}=6$ )

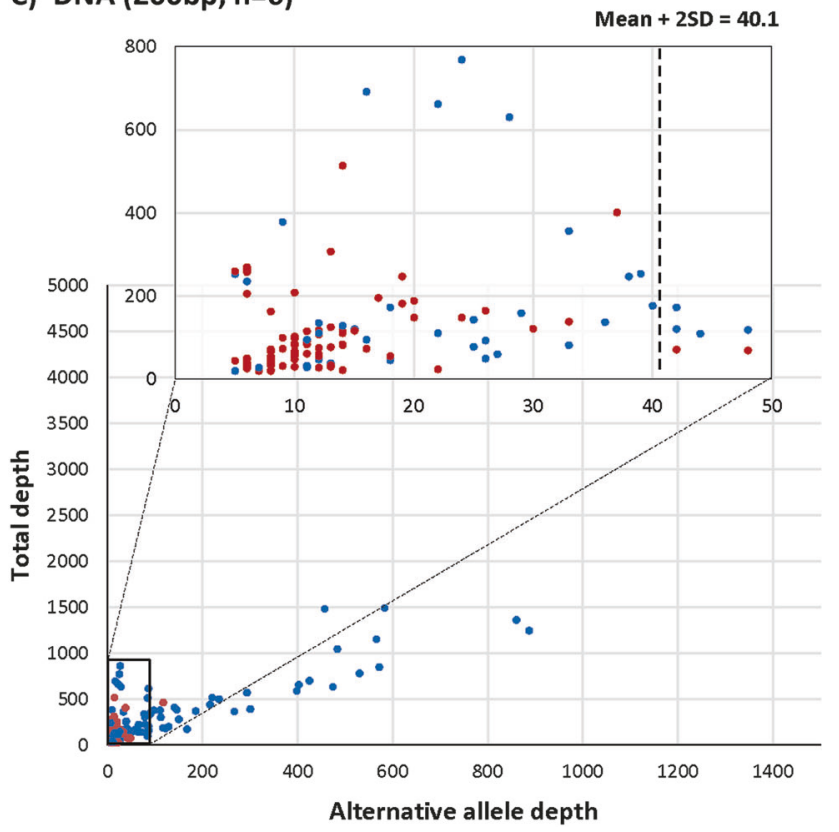

Fig. 2 Alternative allele depth (AAD) as an effective parameter in dichotomy between reproducible and non-reproducible variants. In general, the AAD value for non-reproducible variants is low, particularly in DNA samples that support PCR of $300 \mathrm{bp}$ or more genomic

highly sensitive and specific in mutation screening, comparable to the Fluidigm/Illumina MiSeq approach established previously [6]. This was further supported by the similar mutation frequencies in DLBCL between the present and published studies.
B) DNA (300bp, $\mathrm{n}=21)$

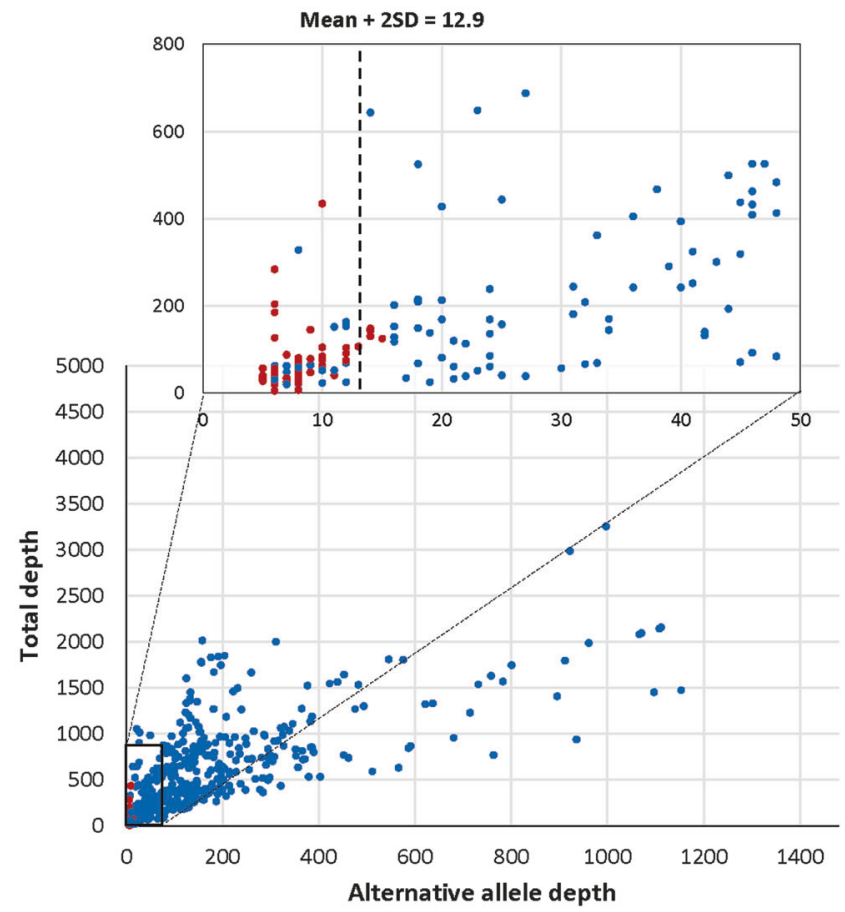

- Reproducible variants

Non-reproducible variants fragments, with mean plus $2 \mathrm{SD}$ being less than 15 AAD. To ensure highly specific mutation detection, a cut-off value of 20 AAD is used for dichotomy between reproducible and non-reproducible variants.

\section{Nature of false positives from FFPE tissue DNA by HaloPlexHS target enrichment}

For non-reproducible changes, $C: G>T: A$ and $C: G>A: T$ alterations accounted for the majority of single substitution 
Fig. 3 Concordance in mutation detection between the HaloPlexHS and Fluidigm target enrichment approach under their respective protocols.
A) Investigated in duplicate by both HaloplexHS and Fluidigm methods $(n=22)$.

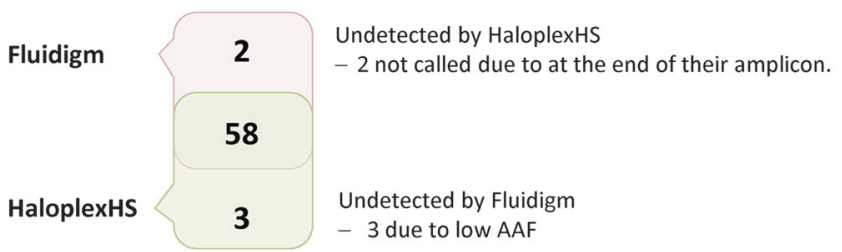

B) Investigated in duplicate by Fluidigm, but single replicate by HaloplexHS method ( $n=38$ )

\begin{tabular}{l|c|c|c|}
$\mathbf{5}$ & $\begin{array}{l}\text { Undetected by HaloplexHS } \\
-2 \text { due to suboptimal AAD; } \\
-1 \text { not called due to at the end of its amplicon } \\
-2 \text { not called due to low AAF }\end{array}$ \\
HaloplexHS & $\mathbf{7}$ & $\begin{array}{l}\text { Undetected by Fluidigm } \\
-7 \text { due to low AAF }\end{array}$
\end{tabular}

changes, with other base changes including A:T>G:C (a feature of PCR errors) being at relatively low frequencies (Fig. S4). In contrast, a wide spectrum of substitution changes was seen for the reproducible changes above the 20 AAD cut-off value. There was no apparent correlation between the type of substitution changes and the quality of DNA samples.

\section{Discussion}

In this study, we have developed a targeted sequencing protocol for mutation screening using HaloPlexHS target enrichment, the Illumina HiSeq platform and an in house variant calling algorithm that was validated against virtual sequence libraries containing a large number of known mutations. By performing the targeted sequencing in duplicate and validating the detected variants against an independent targeted sequencing protocol, namely Fluidigm Access Array PCR and Illumina MiSeq sequencing [6], we have established a stratified approach for mutation screening using DNA samples from FFPE tissues (Fig. 4).

\section{Stratified targeted sequencing approach according to DNA quality}

Under the defined experimental and data analysis protocol and the proposed 20 AAD cut-off value, reliable mutation screening could be achieved by a single replicate for DNA samples amenable for good amplification of $\geq 400 \mathrm{bp}$ genomic fragments. This is evident by the excellent overall sequence coverage, read depth and highly sensitive and specific detection of known mutations in these samples.

For DNA samples showing good amplification of up to $300 \mathrm{bp}$ genomic fragments, reliable mutation detection could still be obtained by a single replicate in the sequence regions that were adequately captured and sequenced. This is evident by the absence of any non-reproducible variants above the 20 AAD cut-off value. However, the overall sequence coverage in these samples is relatively low, with an average of $\sim 8 \%$ (ranging $4-16 \%$ ) targeted sequences not adequately captured and sequenced when only a single replicate was carried out (Fig. 1D). In addition, the extent of non-reproducible variants is much higher than those amenable for PCR of $\geq 400 \mathrm{bp}$ (Fig. 1E). In view of these concerns, these samples are best sequenced in duplicate, which not only improves the sequence coverage albeit moderately, but also enables mutation detection based on reproducible variants, further ensuring the detection specificity (Fig. S5).

For DNA samples that are amplifiable for only up to 200 bp genomic fragments, reliable mutation screening could not be achieved due to poor sequence coverage and substantial numbers of non-reproducible variants above the proposed AAD cut-off value (Figs. 1B-D, 2C). Such samples are best excluded from targeted sequencing using the above experimental conditions. Although it remains to be investigated whether an increase of library quantity from DNA samples of poor quality would improve the sequence coverage, the vast majority of sequence reads in such samples were from re-amplified products (Fig. S2), indicating suboptimal enrichment of the targeted sequences. A more efficient approach to work on DNA samples of poor quality is to redesign the probes by targeting and amplifying smaller sized sequence fragments.

It is important to note that the above AAD cut-off value for dichotomy between reproducible and non-reproducible variants and the proposed stratified targeted sequencing approach according to DNA quality were based on the experimental and data analysis protocols defined in this study. The AAD cut-off value most likely varies depending on the experimental protocol and data analysis pipeline, and thus should be experimentally determined for the methodology to be employed. 


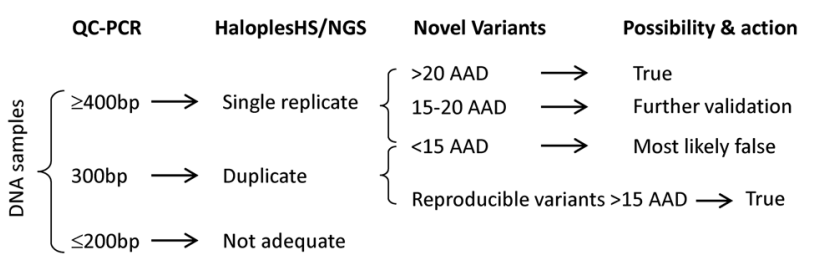

Fig. 4 Proposed stratified approach for mutation screening by HaloplexHS target enrichment and Illumina HiSeq4000 sequencing according to DNA quality. The DNA samples that support PCR of $\geq 400$ bp genomic fragment are amenable for mutation analysis with a single replicate, with only variants at 15-20 AAD required for further validation, while those with suboptimal quality (PCR of up to $300 \mathrm{bp}$ ) are better analysed in duplicate with reproducible variants $>15$ AAD regarded as true genetic changes. DNA samples that support PCR of only 200 bp genomic fragment are not suitable for targeted sequencing under the conditions specified.

Although the cost for NGS is decreasing, targeted sequencing is still costly due to the expensive target enrichment kit and relatively high cost for running fast sequencing platforms such as Illumina MiSeq and HiSeq2500. The above stratified approach would improve the cost effectiveness. Based on our ongoing works, 92\% of DNA samples from FFPE DLBCL tissue biopsies recruited to an ongoing MaPLe clinical trial would be adequate for targeted sequencing using a single replicate. Nonetheless, the proportion of such good DNA samples was relatively lower in the earlier REMoDL-B trial (tissue specimens stored for an average 4 years, range 2-6 years) (87\%) and archival population based DLBCL (tissue specimens stored for an average 9 years, range 5-13 years) (44\%), most likely reflecting variation and deterioration of DNA quality during tissue storage.

\section{Detection of low burden mutations}

As shown in Fig. 2A, B, there was a considerable overlap among the reproducible and non-reproducible variants below the 20 AAD cut-off value, with those below 15 AAD being largely non-reproducible changes. For the variants between 15-20 AAD, it is not possible to ascertain their nature, i.e., true genetic change or false positive if targeted sequencing was performed in a single replicate. Nonetheless, these variants were small in number, and so could be validated by an independent approach such as Sanger sequencing (Fig. 4). A high proportion of these variants could be subclonal genetic changes, and may be beyond the sensitivity of Sanger sequencing detection. Practically, these variants could be amplified by conventional PCR, selectively pooled and barcoded, then sequenced with an Illumina MiSeq platform.

To maximise the sensitivity and specificity of mutation detection, it is critical to use specimens with high tumour cell content for DNA extraction, and where necessary to enrich tumour cell population by microdissection, as this will improve the separation of true genetic changes from background noise. We compared the extent of reproducible variants below 20 AAD according to tumour load, and found that this accounted for only $2 \%$ of the total reproducible variants in DNA samples with $90 \%$ tumour cell content, but $5 \%$ of the total reproducible variants in those with $20-50 \%$ tumour cell content.

\section{Nature of non-reproducible variants}

In the previous study by Fluidigm Access Array PCR and Illumina MiSeq, the vast majority of non-reproducible variants in DNA samples from FFPE tissues were PCR errors (A:T>G:C changes) and changes resulting from cytosine deamination (C:G>T:A) [6]. In contrast to the Fluidigm approach, the present HaloPlexHS protocol showed a low level of A:T>G:C changes among nonreproducible variants, indicating that the incorporation of a molecular barcode in the HaloPlexHS target enrichment design was highly efficient in removing PCR errors. Importantly, this has made it possible for reliable mutation screening by targeted sequencing with a single replicate when the quality of DNA sample is adequate.

In summary, we have established a protocol with defined cut-off values for highly sensitive and specific mutation screening by HaloPlexHS target enrichment and Illumina sequencing, and provided a practical and stratified approach for mutation analysis using DNA samples from FFPE tissues according to their quality. We are currently using this established protocol to perform mutation profiling in DLBCL recruited to the REMoDL-B and MaPLe trials.

Acknowledgements The authors would like to thank Shubha Anand and Yuanxue Huang for their assistance with using TapeStation, and Graeme Clark and Ezequiel Martin for their assistance with Illumina sequencing.

Funding The research was supported by grants from Bloodwise (13006, 15002, 15019) UK, and Kay Kendal Leukaemia Fund (KKL582), UK.

Author contributions Experimental design, data collection and analysis: FC, AC, HK, MW, SB, MVH; Illumina sequencing analysis and variant calling: JST, EOR; Case contribution: SB, MB, JC, DH, TC, $\mathrm{CB}$, AJD, PJ; Manuscript writing and preparation: MQD, FC, HK, JST; Study design and coordination: MQD, PJ, AJD, SB, TC. All authors commented on the manuscript and approve its submission for publication.

\section{Compliance with ethical standards}

Conflict of interest The authors declare that they have no conflict of interest.

\section{References}

1. Do $\mathrm{H}$, Wong SQ, Li J, et al. Reducing sequence artifacts in amplicon-based massively parallel sequencing of formalin-fixed 
paraffin-embedded DNA by enzymatic depletion of uracilcontaining templates. Clin Chem. 2013;59:1376-83.

2. Bourgon R, Lu S, Yan Y, et al. High-throughput detection of clinically relevant mutations in archived tumor samples by multiplexed PCR and next-generation sequencing. Clin Cancer Res. 2014;20:2080-91.

3. Kim S, Park C, Ji Y, et al. Deamination effects in formalin-fixed, paraffin-embedded tissue samples in the era of precision medicine. J Mol Diagn. 2017;19:137-46.

4. Robasky K, Lewis NE, Church GM. The role of replicates for error mitigation in next-generation sequencing. Nat Rev Genet. 2014;15:56-62.

5. Davies KD, Farooqi MS, Gruidl M, et al. Multi-institutional FASTQ file exchange as a means of proficiency testing for nextgeneration sequencing bioinformatics and variant interpretation. J Mol Diagn. 2016;18:572-9.

6. Wang M, Escudero-Ibarz L, Moody S, et al. Somatic mutation screening using archival formalin-fixed, paraffin-embedded tissues by fluidigm multiplex PCR and illumina sequencing. J Mol Diagn. 2015; 17:521-32.

7. Bracho MA, Moya A, Barrio E. Contribution of Taq polymeraseinduced errors to the estimation of RNA virus diversity. J Gen Virol. 1998;79(Pt 12):2921-8.
8. Do H, Dobrovic A. Dramatic reduction of sequence artefacts from DNA isolated from formalin-fixed cancer biopsies by treatment with uracil- DNA glycosylase. Oncotarget. 2012;3:546-58.

9. Hofreiter M, Jaenicke V, Serre D, et al. DNA sequences from multiple amplifications reveal artifacts induced by cytosine deamination in ancient DNA. Nucleic Acids Res. 2001;29: 4793-9.

10. Li H, Handsaker B, Wysoker A, et al. The sequence alignment/ map format and SAMtools. Bioinformatics. 2009;25: 2078-9.

11. DePristo MA, Banks E, Poplin R, et al. A framework for variation discovery and genotyping using next-generation DNA sequencing data. Nat Genet. 2011;43:491-8.

12. Ye K, Schulz MH, Long Q, et al. Pindel: a pattern growth approach to detect break points of large deletions and medium sized insertions from paired-end short reads. Bioinformatics. 2009;25:2865-71.

13. Danecek P, Auton A, Abecasis G, et al. The variant call format and VCFtools. Bioinformatics. 2011;27:2156-8.

14. Quinlan AR, Hall IM. BEDTools: a flexible suite of utilities for comparing genomic features. Bioinformatics. 2010;26: $841-2$. 